\title{
Blocking in All-Optical Networks
}

\author{
Ashwin Sridharan, Student Member, IEEE, and Kumar N. Sivarajan, Member, IEEE
}

\begin{abstract}
We present an analytical technique of very low complexity, using the inclusion-exclusion principle of combinatorics, for the performance evaluation of all-optical, wavelength-division multiplexed networks with no wavelength conversion. The technique is a generalized reduced-load approximation scheme which is applicable to arbitrary topologies and traffic patterns. One of the main issues in computing blocking probabilities in all-optical networks is the significant link load correlation introduced by the wavelength continuity constraint. One of the models we propose takes this into account and gives good results even under conditions with high link load correlation. Through numerous experiments we show that our models can be used to obtain fast and accurate estimates of blocking probabilities in all-optical networks and scale well with the path length and capacity of the network. We also extend one of our models to take into account alternate routing, in the form of Fixed Alternate Routing and Least Loaded Routing.
\end{abstract}

Index Terms-Blocking probability, combinatorial mathematics, optical networks, performance analysis, routing, wavelength division multiplexing.

\section{INTRODUCTION}

W AVELENGTH-DIVISION multiplexing (WDM) is a promising technology which, in conjunction with wavelength routing, is making optical networks with hundreds of nodes and throughput of the order of Gbs/sec per node practical. This is because wavelength routed all- optical networks, in conjunction with WDM, offer wavelength reuse and remove the electro-optic bottleneck. In this work we consider circuit switched all-optical networks since they are a natural outcome of current WDM technology [13]. Circuit (or lightpath) requests arrive at random and are assigned a free wavelength (if available) on each link of the path they use for the duration of the request. A common metric of performance in conventional circuit-switched networks is the call blocking probability, that is, the probability that a call cannot be accepted. Telephony networks set up and tear down voice circuits over the order of minutes which makes blocking probability an important metric. In all-optical networks, lightpaths carry data at speeds of $\mathrm{Gb} / \mathrm{s}$ and are set up or torn down over the order of weeks or months which is a much larger time scale. However, blocking probability can still be a reasonable metric for optical networks. This is because with growing traffic, lightpaths in the US and

Manuscript received March 15, 2000; revised June 17, 2001; approved by IEEE/ACM TRANSACTIONS ON NETwORKING Editor G. Sasaki. This work was supported by a research grant from the Department of Science and Technology, Government of India.

A. Sridharan was with the Indian Institute of Science, Bangalore, India. He is now with the Department of Electrical and Systems Engineering, University of Pennsylvania, Philadelphia, PA 19104 USA (e-mail: ashwin@ee.upenn.edu).

K. N. Sivarajan was with the Indian Institute of Science, Bangalore, India. He is now with Tejas Networks, Bangalore, 560 001, India (e-mail: kumar@ tejasnetworks.com).

Digital Object Identifier 10.1109/TNET.2004.826251
Europe are being leased for varying time durations. Hence, a wavelength is increasingly being viewed as a circuit. There is also a thrust by equipment vendors and service providers in developing standards such as GMPLS [3] which would enable client IP routers to request and tear down lightpaths in the optical core backbone. In this scenario, wavelengths are very similar to circuits. More importantly, the blocking model is still applicable over the order of holding times (though it now takes longer to reach steady state) and blocking probability computations can be used to dimension network and link capacities while taking into account the dynamics of lightpath requests. These observations suggest that blocking probability is still a useful metric in analysing the performance of optical networks.

In the context of assigning a wavelength to the lightpath request, the two different functional aspects of wavelength routing come into play. If the nodes have wavelength conversion capability, the request can be assigned different wavelengths on each link of the path used. In such a situation, the all-optical network reduces to a standard circuit switched network. However, if the nodes cannot do wavelength conversion, the request must be assigned the same wavelength on all the links of the path used. This is known as the wavelength continuity constraint and makes networking in the all-optical domain significantly different from conventional circuit switched networks. Clearly, networks with wavelength changers have a lower blocking probability compared to those without because they only require some wavelength (which can be different) to be free on each link of the path, whereas networks without changers require the same wavelength to be free on all the links of a path in order to honor a request. Hence, it would be desirable to have wavelength converters on every node in a network. However, wavelength converters are still in the experimental stage and are likely to remain very expensive, if implemented. Hence, it is important to quantify the call blocking performance of optical networks without wavelength conversion.

Research has shown that the wavelength continuity constraint introduces load correlation between links, and that the blocking in the network is affected by the type of wavelength assignment and routing scheme used. Models for the first fit wavelength assignment scheme have been proposed in [6]-[8]. They, however, use versions of the overflow traffic model and are applicable only when the number of wavelengths are small. Various schemes that combine the wavelength assignment and routing problem have been proposed and studied, [2], [5], [8], [10] and [11] being a few in our notice. The reader is referred to [7] for a review of these schemes and their effectiveness. A bound on the carried traffic in an arbitrary network by any RWA algorithm was derived in [14]. The bound is, however, only asymptotically achievable. 
Analytical models for analyzing the performance of optical networks with random wavelength assignment and no wavelength changers have been proposed in [1], [2], [16], [18]-[20]. In [1], Barry et al. proposed an analytical model to study the effectiveness of wavelength changers, taking wavelength correlation into account. However, the model does not take into account the dynamic nature of the traffic. The model proposed by Subramanium et al. [16] takes both dynamic traffic and wavelength correlation into account and has been shown to be accurate even for sparse networks like rings. Moreover, the model has a moderate complexity. It is, however, applicable in the strict sense only to networks with uniform traffic and regular topologies. In case of irregular topologies and traffic distributions, only ensembles are used. Another model proposed by Birman [2] uses a reduced load approximation approach along with state-dependent arrival rates. The model is shown to be good for small networks where multilink traffic is not appreciable and is applicable to arbitrary topologies and traffic patterns. The model has also been extended to incorporate Fixed Alternate Routing (FAR) and Least Loaded Routing (LLR). It is, however, computation intensive, with the complexity growing exponentially with the number of hops and does not take load correlation introduced by the wavelength continuity constraint into account. Hence, it is tractable only for small dense networks. A model proposed in [19] by Zhu et al. and further developed in [20] and [18] uses a modified time reversible Markov chain to account for link correlation. The model is accurate and applicable to arbitrary topologies and traffic patterns. However, the models we develop in this paper are simpler with lower computation overhead while still yielding good quality results. The reader is referred to [12] and [7] for a review of these analytical models.

Future wide area networks are most likely to be all-optical networks with tens, if not hundreds of nodes, connected in an arbitrary fashion with a large number of wavelengths per fiber. Hence, we require a technique applicable to arbitrary topologies which is computationally tractable, and also gives reasonable estimates of blocking probabilities for design purposes and the analytical study of issues like benefits of wavelength changers, alternate routing etc. With this goal in mind we propose two techniques in this paper which have a very low complexity of calculation and are applicable to arbitrary topologies and traffic patterns. The models scale very well with path length or capacity of a link (in number of wavelengths) and use only simple computations while giving remarkably accurate results. In order to reduce the complexity of computations, we have used the inclusion-exclusion principle from combinatorics to obtain simple expressions for path blocking and improve their accuracy by incorporating some simple heuristics. We use the reduced load approximation scheme ([4]) to account for arbitrary traffic patterns and topologies. The first technique makes the link independence assumption and is called the Independence Model. Estimates from this model are reasonable when the network is dense or end-to-end traffic is negligible, but overestimates significantly when the multihop lightpaths have appreciable traffic and the network is sparse. In fact, we show that the estimates are same as those obtained in [2], but without the bottleneck of exponential complexity (the complexity scales polynomially only with the capacity of the link). To account for wavelength correlation we propose another model which we call, naturally, the Correlation Model. We show that this model is accurate even for sparse networks which have significant link load correlation. We then extend both models in order to analyze FAR and LLR.

The rest of the paper is organized as follows. In Section II we outline the basic network and traffic assumptions used throughout the paper. We present the Independence Model in Section III and the Correlation Model in Section IV. We then use them to analyze the FAR scheme in Section V and LLR in Section VI. We present our results in Section VII and conclude in Section VIII.

\section{Network AND TRAFFIC MODEL ASSUMPTIONS}

In this section we state the assumptions about the network and traffic that are used in the model for calculating the path blocking probability of an optical network with no wavelength changers. All assumptions stated here are valid throughout the paper.

\section{A. Assumptions Regarding the Network and Offered Traffic}

1) The network consists of $J$ links connected in an arbitrary fashion.

2) Each link has the same $C$ wavelengths.

3) Calls for a node pair $S$ arrive according to a Poisson with rate $\lambda_{S}$.

4) The duration of each call is exponentially distributed with unit mean.

5) A call can be accommodated on a route only if the same wavelength is free on all the routes. That is, we assume that the optical network does not have anywavelength changers.

6) The wavelength assigned to a route is chosen randomly from the set of free wavelengths. This assumption makes all wavelengths identical and the analysis tractable.

7) We assume that wavelength occupancy on disjoint routes are independent of each other.

We now justify some of our assumptions. As mentioned previously, the practical utility of blocking probability computations lies in using them to dimension network/link capacities. This usually requires repeated blocking probability computations. Hence, we need a model that is not only accurate but fast too. Simulations provide more accurate results but are too time consuming to be of practical use for large networks with large capacities. In the same context, the impact of wavelength assignment algorithms on network dimensioning is not significant. Hence, a wavelength assignment that is more amenable to analysis would be better suited than "superior" wavelength assignments, like first-fit, which are analyzed through simulations. After the network is dimensioned, one could still use the superior wavelength assignment algorithms during operation. This is the same reason we make assumptions about the arrival traffic pattern and holding times. They make the problem amenable to analysis and provide a reasonably good estimate of blocking probability for network dimensioning even though actual traffic may not share the same statistical properties. 


\section{B. Traffic Model}

We assume that the idle wavelength distribution on a link can be described by the state-dependent routing model first proposed in [9] and developed in [17]. The same model is also used in [2]. Below, we describe the model and its assumptions. Let $Z_{R}$ be the random variable representing the number of free wavelengths on route $R$.

Let $X_{j}$ be the random variable representing the number of free wavelengths on link $j$. Let

$$
q_{j}(m)=\operatorname{Pr}\left\{X_{j}=m\right\}
$$

be the probability that exactly $m$ wavelengths are free on link $j$. The random variables $X_{j}$ are assumed to be independent, that is

$$
q(\mathbf{m})=\prod_{j=1}^{J} q_{j}\left(m_{j}\right) .
$$

Following [17] we assume that, given exactly $m$ idle wavelengths on link $j$, the time until the next call setup on $j$ is exponentially distributed with parameter $\alpha_{j}(m)$. It then follows that the number of idle wavelengths on link $j$ can be thought of as a birth-death process. We then have

$$
q_{j}(m)=\frac{C(C-1) \ldots(C-m+1)}{\alpha_{j}(1) \alpha_{j}(2) \ldots \alpha_{j}(m)} q_{j}(0)
$$

where

$$
q_{j}(0)=\left[1+\sum_{m=1}^{C} \frac{C(C-1) \ldots(C-m+1)}{\alpha_{j}(1) \alpha_{j}(2) \ldots \alpha_{j}(m)}\right]^{-1} .
$$

The call set-up rate is a function of the routing scheme used. We assume fixed routing in this section and Sections III and IV also. This means that each node pair has exactly one pre-determined route. If an arriving call does not find a free wavelength on this route, it is blocked and lost.

For fixed routing the call setup rate when there are exactly $m$ idle wavelengths on link $j$ is obtained by combining the contributions from all the request streams that have link $j$ as their member. This expression was first obtained for an all-optical network in [2].

$$
\begin{aligned}
\alpha_{j}(m) & =0 \text { if } m=0 \\
& =\sum_{R: j \in R} \lambda_{R} \operatorname{Pr}\left\{Z_{R}>0 \mid X_{j}=m\right\} \\
m & =1,2, \ldots, C .
\end{aligned}
$$

Typically, in a network, the blocking probabilities and arrival rates to a link are coupled to each other by the fact that the blocking determines the traffic carried by the network and the carried traffic in turn determines the blocking. This leads to a set of coupled nonlinear equations which must be solved to obtain the blocking probabilities. The usual scheme implemented in most analyzes is solution by iteration and is the one used in this paper too.

\section{INDEPENDENCE MODEL}

In this section we present the technique for calculating the blocking probability along a path from the link distributions.
The probability that a call traversing a route $R$ consisting of a single link $j$ (say) is blocked is simply given by

$$
B_{R}=q_{j}(0)
$$

which is the probability that there is no idle wavelength on link $j$.

In order to calculate the blocking probability for a multihop path we introduce the following random variable:

Let $Y_{i, j}$ be the random variable denoting the state of wavelength $i$ on link $j$.

Define

$$
\begin{aligned}
& Y_{i, j}=0, \text { if wavelength } i \text { is free on link } j, \\
& Y_{i, j}=1, \text { if wavelength } i \text { is used on link } j .
\end{aligned}
$$

From the assumption of random wavelength assignment, we then have the probability that a fixed set of $i$ wavelengths is free on link $j$ given by

$$
\operatorname{Pr}\left\{Y_{1, j}=0, \ldots, Y_{i, j}=0\right\}=\sum_{m=i}^{C} q_{j}(m) \frac{\left(\begin{array}{c}
m \\
i
\end{array}\right)}{\left(\begin{array}{c}
C \\
i
\end{array}\right)} .
$$

This may be seen as follows. Since we assume random wavelength assignment, given exactly $m$ wavelengths are free, each of the possible set of $m$ free wavelengths can occur (be chosen) with equal probability, which is $\left(\begin{array}{c}C \\ m\end{array}\right)^{-1}$. Now, we require that our fixed set of $i$ wavelengths be among the set of $m$ free wavelengths. This implies that the remaining $m-i$ wavelengths must be chosen from $C-i$ wavelengths, which can happen in $\left(\begin{array}{c}C-i \\ m-i\end{array}\right)$ ways. Hence, the probability that, given exactly $m$ wavelengths are free, a fixed set of $i$ wavelengths is among them, is

$$
\left(\begin{array}{c}
C-i \\
m-i
\end{array}\right)\left(\begin{array}{c}
C \\
m
\end{array}\right)^{-1}
$$

On simplifying, this reduces to, $\left(\begin{array}{c}m \\ i\end{array}\right)\left(\begin{array}{c}C \\ i\end{array}\right)^{-1}$.

We denote by

$$
\beta_{i, j}=\operatorname{Pr}\left\{Y_{k_{1}, j}=0, Y_{k_{2}, j}=0, \ldots, Y_{k_{i}, j}=0\right\}
$$

the probability that a fixed set of $i$ wavelengths is free on link $j$. Note that since the wavelengths are identical by virtue of the assumption of random assignment, the actual subscript $k_{l}$ in $Y_{k_{l}, j}$ does not matter. All we require is that the set contain $i$ wavelengths. In other words, all sets of $i$ wavelengths are equally likely to be free.

Now, the probability that a multihop route $R$ (say) is blocked is the probability that there is no wavelength which is free on all the links used by $R$. We have

$$
B_{R}=\operatorname{Pr}\left\{Z_{R}=0\right\}=1-\operatorname{Pr}\left\{Z_{R}>0\right\} .
$$

Let $g_{i}$ be the probability that a fixed set of $i$ wavelengths is free on the route $R$. Then, from the inclusion-exclusion principle and 
the assumption of random wavelength assignment, it follows that

$$
\operatorname{Pr}\left\{Z_{R}>0\right\}=\sum_{i=1}^{C}(-1)^{i-1}\left(\begin{array}{c}
C \\
j
\end{array}\right) g_{i} .
$$

For notational convenience we describe in detail the method of calculation of $g_{i}$ for a two-link path consisting of links $A$ and $B$. The method is readily generalized for paths with higher hop lengths.

For the two-link path

$$
\begin{aligned}
& g_{i}=\operatorname{Pr}\left\{\left(Y_{1, A}=0, Y_{1, B}=0\right),\right. \\
&\left.\left(Y_{2, A}=0, Y_{2, B}=0\right), \ldots,\left(Y_{i, A}=0, Y_{i, B}=0\right)\right\} .
\end{aligned}
$$

Using the assumption that the sets of wavelengths on links are independent (1), we have

$$
\begin{aligned}
g_{i}=\operatorname{Pr}\left\{Y_{1, A}=0, Y_{2, A}=0, \ldots, Y_{i, A}=0\right\} & \\
\cdot & \operatorname{Pr}\left\{Y_{1, B}=0, Y_{2, B}=0, \ldots, Y_{i, B}=0\right\}
\end{aligned}
$$

or, from (7)

$$
g_{i}=\beta_{i, A} \cdot \beta_{i, B}
$$

The method described above immediately generalizes to higher hop lengths. The probability that a call is blocked on a $H$-hop route $R$ is given by

$$
B_{R}=\operatorname{Pr}\left\{Z_{R}=0\right\}=1-\operatorname{Pr}\left\{Z_{R}>0\right\}
$$

where

$$
\operatorname{Pr}\left\{Z_{R}>0\right\}=\sum_{i=1}^{C}(-1)^{i-1}\left(\begin{array}{c}
C \\
i
\end{array}\right) g_{i}
$$

and $g_{i}$ is given by

$$
g_{i}=\prod_{j: j \in R} \beta_{i, j}
$$

\section{A. Calculation of State-Dependent Arrival Rates}

The other part of the blocking computation is to derive the arrival rate of traffic at a link $j$ given $m$ wavelengths are idle, that is $\alpha_{j}(m)$. The arrival rate of a request stream from route $R$ to link $j$, given that there are $m$ wavelengths free on link $j$, is given by (4) as

$$
\begin{aligned}
\alpha_{j}^{R}(m) & =0 \text { if } m=0 \\
& =\lambda_{R} \cdot \operatorname{Pr}\left\{Z_{R}>0 \mid X_{j}=m\right\} \quad m=1,2 \ldots C
\end{aligned}
$$

where

$$
\alpha_{j}(m)=\sum_{j: j \in R} \alpha_{j}^{R}(m) .
$$

If the route consists of a single link then the probability term is clearly 1 for $m \neq 0$. The term for a multihop path may be calculated as follows:

$$
\operatorname{Pr}\left\{Z_{R}>0 \mid X_{j}=m\right\}=\sum_{i=1}^{m}(-1)^{i-1}\left(\begin{array}{c}
C \\
i
\end{array}\right) g_{i}\left(X_{j}=m\right)
$$

where $g_{i}\left(X_{j}=m\right)$ is the conditional probability that a set of $i$ wavelengths is free on the route $R$ given exactly $m$ wavelengths are free on link $j$. It may be calculated as

$$
g_{i}\left(X_{j}=m\right)=\prod_{k: k \in R, k \neq j} \beta_{i, k} \frac{\left(\begin{array}{c}
m \\
i
\end{array}\right)}{\left(\begin{array}{c}
C \\
i
\end{array}\right)}
$$

because

$$
\operatorname{Pr}\left\{Y_{1, j}=0, \ldots, Y_{i, j}=0 \mid X_{j}=m\right\}=\frac{\left(\begin{array}{c}
m \\
i
\end{array}\right)}{\left(\begin{array}{c}
C \\
i
\end{array}\right)} .
$$

Note that the summation in (11) runs only up to $m$ since $m$ is an upper bound on the number of free wavelengths on the path.

\section{B. Algorithm for Computation of Blocking Probabilities in the Network}

As mentioned in Section II, we need to solve a set of nonlinear coupled equations to obtain the blocking probabilities. Though it is not clear whether a fixed point exists for this system of coupled equations (and if it does whether it is unique), in practice the method of solution by repeated substitution converges in a few iterations for a variety of topologies. The method of repeated substitution to solve for the blocking probabilities may be implemented as follows:

Let $B_{R}$ be the probability that a call for route $R$ is blocked.

1) For all routes $R$ initialize $\hat{B_{R}}$ to zero. For $j=1, \ldots, J$ initialize $\alpha_{j}(0)=0$ and $\alpha_{j}(m)=\sum_{R: j \in R} \lambda_{R}, m=$ $1, \ldots C$.

2) Determine the idle capacity distribution of all links $q_{j}(\cdot)$, $j=1, \ldots, J$ using (2) and (3).

3) Calculate $\beta_{j, m}$ for all links, $j=1, \ldots, J$ and $m=$ $1, \ldots, C$ using (6).

4) Calculate $\alpha_{j}(\cdot), j=1, \ldots, J$ using (4), (11) and (12).

5) Calculate $B_{R}$ for all routes using (5) if it is a single link and (8)-(10) for a multihop path.

If $\max _{R}\left|B_{R}-\hat{B_{R}}\right|<\epsilon$ then terminate. Else let $\hat{B_{R}}=B_{R}$, go to step 2 .

\section{CORRELATION MOdel}

As will be shown in Section VII the independence model presented in Section III gives good results for dense networks but overestimates the blocking probability significantly for sparse networks like rings. This is because it does not account for load correlation introduced by the wavelength continuity constraint between adjacent links. That is, it assumes that states of sets of wavelengths on adjacent links are independent, which is clearly not true when the network is sparse. In sparse networks like rings, the number of choices for a route is small. Hence, calls tend to stay together over a longer set of links leading to increased correlation since they use the same wavelength on all the links.

In this section we extend the independence model to take this correlation into account. The tradeoff for accuracy, however, is 
that the complexity of calculation increases. But as will be seen, it is still very amenable to computations.

The network and traffic assumptions made in Section II remain the same. All the notations and variables used in Section III retain their original meanings. We also assume that the parameters $\beta_{i, j}$ may be calculated as before, using (6). The point of departure from the previous model is that we no longer make the assumption of unconditional wavelength independence made previously while calculating the blocking on a multihop path $R$. Instead we make a set of weaker assumptions as described below:

- A1. The state of a wavelength $i$ on link $j$ is independent of the state of some other wavelength $k$ on link $j-1$, given the state of the same wavelength $i$ on link $j-1$, or the state of wavelength $k$ on the same link $j$.

More formally

$Y_{i, j}$ is independent of $Y_{k, j-1} k \neq i$ given $Y_{k, j}$ or $Y_{i, j-1}$.

The intuition behind our assumption is as follows. Because of the wavelength continuity constraint, if there is significant multihop traffic, the state of a wavelength $i$ on some link $j$ is more likely to be affected by the state of the same wavelength on a previous link than any other wavelength on the previous link. Similarly, if the amount of multihop traffic is not significant, links can be treated independently and the state of wavelength $i$ on a link $j$ is more likely to be affected by the state of the wavelengths on the same link $j$. By combining these two cases we obtain assumption A1 which tries to cater for cases where there is significant link load correlation and also cases where links can be treated independently.

- A2. On a given route, the state of a wavelength on a link $j$ is independent of the state of the same wavelength on previous or successive links of the route, given the state of the wavelength on link $j-1$. More formally

$$
Y_{i, j} \text { is independent of } Y_{i, k} j \neq k \text { given } Y_{i, j-1} \text {. }
$$

Assumption A2 is based on the intuitive idea of locality, that is, a wavelength on some link $j$ is more likely to be affected by the state of the wavelength on links neighboring $j$ than links than are far away. By using A2, we can compute the blocking of multihop paths iteratively.

The probability of blocking on a multihop route $R$ is given as before by

$$
\operatorname{Pr}\left\{Z_{R}=0\right\}=1-\operatorname{Pr}\left\{Z_{R}>0\right\}
$$

where

$$
\operatorname{Pr}\left\{Z_{R}>0\right\}=\operatorname{Pr}\left\{Z_{R}>0\right\}=\sum_{i=1}^{C}(-1)^{i-1}\left(\begin{array}{c}
C \\
i
\end{array}\right) g_{i}
$$

and the $g_{i}$ 's retain their usual meaning.

We now depart from the technique in Section III in that we derive a new expression for calculating $g_{i}$.

For clarity of exposition we shall first derive the expression for $g_{i}$ on a two-link path. The expressions can be easily gener- alized for higher hop-length paths. Consider a two-link path $R$ (say) over links $A$ and $B$. We have

$$
\begin{aligned}
g_{i}=\operatorname{Pr}\left\{\left(Y_{1, A}=0, Y_{1, B}=0\right), \ldots,\right. \\
\left.\left(Y_{2, A}=0, Y_{2, B}=0\right),\left(Y_{i, A}=0, Y_{i, B}=0\right)\right\} .
\end{aligned}
$$

Using the chain rule and assumption (A1) this may be simplified to

$$
\begin{array}{r}
g_{i}=\operatorname{Pr}\left\{Y_{i, A}=0 \mid Y_{i-1, A}=0 \ldots Y_{1, A}=0, Y_{i, B}=0\right\}, \ldots \\
\operatorname{Pr}\left\{Y_{1, A}=0 \mid Y_{1, B}=0\right\} \cdot \beta_{i, B} .
\end{array}
$$

The term $\operatorname{Pr}\left\{Y_{i, A}=0 \mid Y_{i-1, A}=0 \ldots Y_{1, A}=0, Y_{i, B}=0\right\}$ can be further simplified as follows.

Define the following new variables

$$
\gamma_{j, j-1}^{(0)}=\operatorname{Pr}\left\{Y_{i, j}=0 \mid Y_{i, j-1}=0\right\}
$$

and

$$
\gamma_{j, j-1}^{(1)}=\operatorname{Pr}\left\{Y_{i, j}=0 \mid Y_{i, j-1}=1\right\} .
$$

Note that the $\gamma_{j, j-1}^{(\cdot)}$ 's do not depend on the wavelength index $i$ since all wavelengths are identical.

Also define

$$
\begin{aligned}
\eta_{i, j} & =\beta_{i, j} \quad \text { if } i=1 \\
& =\frac{\beta_{i, j}}{\beta_{i-1, j}} \quad \text { otherwise. }
\end{aligned}
$$

Observe that $\eta_{i, j}$ is the conditional probability of wavelength $i$ being free given that $i-1$ other wavelengths are free. That is

$$
\eta_{i, j}=\operatorname{Pr}\left\{Y_{i, j}=0 \mid Y_{1, j}=0, Y_{2, j}=0, \ldots, Y_{i-1, j}=0\right\} .
$$

The above-defined terms along with assumption (A1) and Bayes rule allows us to write, after some manipulation,

$$
\begin{aligned}
\operatorname{Pr}\left\{Y_{i, A}=0 \mid Y_{i-1, A}\right. & \left.=0 \ldots Y_{1, A}=0, Y_{i, B}=0\right\} \\
& =\frac{\gamma_{B, A}^{(0)} \eta_{i, A}}{\gamma_{B, A}^{(0)} \eta_{i, A}+\gamma_{B, A}^{(1)} \cdot\left(1-\eta_{i, A}\right)}
\end{aligned}
$$

Substituting (17) in (13) yields

$$
g_{i}=\prod_{k=1}^{i} \frac{\gamma_{B, A}^{(0)} \eta_{k, A}}{\gamma_{B, A}^{(0)} \eta_{k, A}+\gamma_{B, A}^{(1)} \cdot\left(1-\eta_{k, A}\right)} \cdot \beta_{i, B} .
$$

\section{A. Estimation of the Correlation Coefficients}

We have introduced two new parameters $\gamma_{j, j-1}^{(0)}$ and $\gamma_{j, j-1}^{(1)}$ which characterize the load correlation between two adjacent links. The same correlation coefficients were first obtained in [1] and derived again in [15]. We now propose a method to calculate these coefficients for arbitrary topologies and under general traffic patterns.

Let $P_{l}^{(j)}$ be the probability that a session occupying wavelength $\lambda$ on link $j$ does not continue to link $j+1$.

Define a new call on $j$ to be one which does not pass through link $j-1$. Let $P_{n}^{(j)}$ be the probability that a new call occupies wavelength $\lambda$ on link $j$. Then

$$
\begin{aligned}
& \gamma_{j, j-1}^{(0)}=\operatorname{Pr}\left\{Y_{\lambda, j}=0 \mid Y_{\lambda, j-1}=0\right\}=\left(1-P_{n}^{(j)}\right) \\
& \gamma_{j, j-1}^{(1)}=\operatorname{Pr}\left\{Y_{\lambda, j}=0 \mid Y_{\lambda, j-1}=1\right\}=P_{l}^{(j-1)}\left(1-P_{n}^{(j)}\right) .
\end{aligned}
$$


This may be explained as follows. $\gamma_{j, j-1}^{(0)}$ is the probability that wavelength $\lambda$ is free on link $j$ given that it is free on link $j-1$. This is simply the probability that no new call arrives on wavelength $\lambda$ on link $j$ which by definition is $1-P_{n}^{(j)} \cdot \gamma_{j, j-1}^{(1)}$ is the probability that wavelength $\lambda$ is free on link $j$ given that it is used on $j-1$. This is the probability that wavelength $\lambda$ is used on $j-1$ by a session that does not continue to link $j$ and that no new call arrives on wavelength $\lambda$ on link $j$ which is $P_{l}^{(j-1)}\left(1-P_{n}^{(j)}\right)$. We note that we have made use of assumption A2 in deriving these equations, whereby, we have eliminated dependence of link $j$ on all links but $j-1$.

Hence, the correlation coefficients are actually $P_{l}^{(j)}$ and $P_{n}^{(j)}$ which are then substituted in a suitable form to obtain the final expression for $g_{i}$.

$P_{l}^{(j)}$ may be calculated as

$$
P_{l}^{(j)}=\frac{\tilde{\lambda}(j, j+1)}{\tilde{\lambda}_{j}}
$$

where

$$
\tilde{\lambda}_{j}=\sum_{m=1}^{C} \alpha_{j}(m) \cdot q_{j}(m)
$$

is the average arrival rate of traffic to link $j$ and

$$
\tilde{\lambda}(j, k)=\sum_{\substack{\mathbf{R}: j \in R \\ k \notin R}} \sum_{m=1}^{C} \alpha_{j}^{R}(m) \cdot q_{j}(m)
$$

is the rate of accepted traffic which passes through link $j$ but does not pass through link $k$.

Thus, we model $P_{l}^{(j)}$ as the ratio of arrival rate of traffic to link $j$ that does not continue to link $j+1$ to the total traffic arriving at link $j$ which is a reasonable approximation. We calculate $P_{n}^{(j)}$ as follows. Let $\rho_{j}$ be the probability that a wavelength is busy on link $j$ i.e., $\rho_{j}$ is a measure of wavelength utilization of link $j$. Then

$$
P_{n}^{(j)}=\rho_{j} \frac{\tilde{\lambda}(j, j-1)}{\tilde{\lambda}_{j}}
$$

where $\rho_{j}$ is given by

$$
\rho_{i}=1-\operatorname{Pr}\left\{Y_{1, j}=0\right\}=1-\beta_{1, j} .
$$

Hence, $P_{n}^{(j)}$ is the probability that the wavelength is in use on link $j$ and the session using it is one that arrives without passing through link $j-1$, i.e., a new session.

Substituting for $\gamma_{j, j-1}^{(0)}$ and $\gamma_{j, j-1}^{(1)}$ from (19) and (20), the expression for $g_{i}$ may be written as

$$
g_{i}=\prod_{k=1}^{i} \frac{\eta_{k, A}}{\eta_{k, A}+P_{l}^{(A)}\left(1-\eta_{k, A}\right)} \beta_{i, B} .
$$

Observe that if we put $P_{l}^{(A)}=1, g_{i}$ reduces to the expression obtained in the Independence model presented in Section III $i$ which is correct. Again, if $P_{l}^{(A)} \rightarrow 0$, (negligible single-link traffic) the expression reduces to $\beta_{i, B}$, which is also correct. Hence, we expect the Correlation Model to perform well under different patterns of traffic, an observation confirmed in Section VII. Also observe that the final expression for $g_{i}$ after substituting for $\gamma_{j, j-1}^{(0)}$ and $\gamma_{j, j-1}^{(1)}$ is independent of $P_{n}^{(j)}$ ! This comes about because we make the assumption that the probability that a new call occupies wavelength $\lambda$ on link $j$ is independent of the state of the wavelength on link $j-1$, that is, $P_{n}^{(j)}$ is independent of $P_{l}^{(j)}$.

The expression for blocking probability can now be easily generalized to higher hop paths. For symbolic convenience, let the links on the path be numbered $1,2, \ldots, H$.

Only the expression for $g_{i}$ need be modified. This is done as follows. We make use of assumption (A2) to divide the path into two link subsections and proceed as before for each two link subsection to obtain the probability of blocking on a multihop path as

$$
\operatorname{Pr}\left\{Z_{R}=0\right\}=1-\operatorname{Pr}\left\{Z_{R}>0\right\}
$$

and

$$
\operatorname{Pr}\left\{Z_{R}>0\right\}=\sum_{i=1}^{C}(-1)^{i-1}\left(\begin{array}{c}
C \\
i
\end{array}\right) g_{i}
$$

where

$$
g_{i}=\prod_{j=1}^{H-1} \prod_{k=1}^{i} \frac{\eta_{k, j}}{\eta_{k, j}+P_{l}^{(j)} \cdot\left(1-\eta_{k, j}\right)} \beta_{i, H} .
$$

\section{B. Calculation of State-Dependent Arrival Rates}

Recall from Section II, that in order to calculate state-dependent arrival rates, we need to calculate the probabilities

$$
\operatorname{Pr}\left\{Z_{R}>0 \mid X_{j}=m\right\}=\sum_{i=1}^{m}(-1)^{i-1}\left(\begin{array}{c}
C \\
i
\end{array}\right) g_{i}\left(X_{j}=m\right)
$$

where $g_{i}\left(X_{j}=m\right)$ is the conditional probability that a set of $i$ wavelengths is free on the route $R$ given exactly $m$ wavelengths are free on link $j$. This may be easily calculated by splitting the path into three independent subsections, the path consisting of links before $j$, link $j$, and the path consisting of links after $j$.

Then, $g_{i}\left(X_{j}=m\right)$ is modified to

$$
\begin{aligned}
& g_{i}\left(X_{j}=m\right)=\prod_{\substack{n=1 \\
n \neq j}}^{H-1} \prod_{k=1}^{i} \frac{\eta_{k, n}}{\eta_{k, n}+P_{l}^{(n)}\left(1-\eta_{k, n}\right)} \cdot \beta_{i, H} \frac{\left(\begin{array}{c}
m \\
i
\end{array}\right)}{\left(\begin{array}{c}
C \\
i
\end{array}\right)} \\
& \text { if } j \neq H \\
& \prod_{n=1}^{H-1} \prod_{k=1}^{i} \frac{\eta_{k, n}}{\eta_{k, n}+P_{l}^{(n)}\left(1-\eta_{k, n}\right)} \cdot \frac{\left(\begin{array}{c}
m \\
i
\end{array}\right)}{\left(\begin{array}{c}
C \\
i
\end{array}\right)} \\
& \text { if } j=H
\end{aligned}
$$

where all symbols retain their usual meaning. Note that the summation in (30) runs only up to $m$ because $m$ is an upper bound on the number of free wavelengths on the path. The algorithm for calculating blocking in a network using the Correlation Model is similar to that given in Section III and, hence, we omit it. 


\section{Fixed Alternate Routing}

So far we have assumed that a call can be routed only on a single pre-determined route. However, a good routing scheme can reduce blocking and improve flexibility without addition in hardware costs. We now analyze two popular routing schemes which allow for a call to be assigned on an alternate route, namely, Fixed Alternate Routing (FAR) and Least Loaded Routing (LLR). Both share a common feature in that each node pair is allowed a pre-determined set of possible alternate routes for call set up and differ only in the manner in which the routing algorithm selects the alternate route. Both schemes have their roots in conventional circuit-switched networks, wherein, trunk reservation was used to remove bi-stability. This means that a call can be set up on an alternate route only if a minimum number of wavelengths (circuits) (called the reservation parameter) are free. Suppose that the reservation parameter on the route is $r$. Then the route must have at least $r+1$ wavelengths free before a call can be set up on it.

In order to analyze such schemes, we need to calculate the probability that exactly $l$ wavelengths are free on a given route.

We do so, by introducing a new variable. Let $h_{k}$ denote the probability that exactly a fixed set of $k$ wavelengths is free on a given route $R$. All our following arguments pertain to a specific route $R$ and, hence, we have dropped the subscript for clearer exposition. Observe that all the $h_{k}$ 's refer to disjoint events. Then clearly

$$
\sum_{k=0}^{C}\left(\begin{array}{l}
C \\
k
\end{array}\right) h_{k}=1
$$

where $h_{0}$ is the probability that no wavelength is free on the route and the probability that exactly $l$ wavelengths are free on the route $R$ is simply

$$
\operatorname{Pr}\left\{Z_{R}=l\right\}=\left(\begin{array}{c}
C \\
l
\end{array}\right) h_{l} .
$$

Using combinatorial arguments, we may express $g_{k}, k=1$, $\ldots, C$ in terms of $h_{k}, k=1, \ldots, C$ as

$$
g_{k}=\sum_{i=0}^{C-k}\left(\begin{array}{c}
C-k \\
i
\end{array}\right) h_{k+i} \quad k=1, \ldots, C
$$

where the $g_{k}$ 's retain their original meaning, i.e., it is the probability that a fixed set of wavelengths is free on the route $R$. Solving the above set of equations for $h_{k}, k=1, \ldots, C$ we obtain

$$
h_{k}=\sum_{i=0}^{C-k}(-1)^{i}\left(\begin{array}{c}
C-k \\
i
\end{array}\right) g_{k+i} \quad k=1, \ldots, C .
$$

The above procedure can be easily extended to the case where exactly $m$ wavelengths are free on link $j$ of the route $R$.

Let $h_{k}\left(X_{j}=m\right)$ be the probability that exactly a fixed set of $k$ wavelengths is free on some given route $R$, given exactly $m$ wavelengths are free on link $j$. Then

$$
\sum_{k=0}^{m}\left(\begin{array}{l}
C \\
k
\end{array}\right) h_{k}\left(X_{j}=m\right)=1
$$

and

$$
\begin{aligned}
g_{k}\left(X_{j}=m\right) & =\sum_{i=0}^{m}\left(\begin{array}{c}
C-k \\
i
\end{array}\right) h_{k+i}\left(X_{j}=m_{j}\right), \\
k & =1, \ldots, m_{j} .
\end{aligned}
$$

Solving the above set of equations for $h_{k}\left(X_{j}=m\right), k=1$, ..., $m$ we obtain

$$
\begin{aligned}
h_{k}\left(X_{j}=m\right) & =\sum_{i=0}^{m-k}(-1)^{i}\left(\begin{array}{c}
C-k \\
i
\end{array}\right) g_{k+i}\left(X_{j}=m\right), \\
k & =1, \ldots, m .
\end{aligned}
$$

The probability that exactly $l$ wavelengths are free on route $R$ given exactly $m$ wavelengths are free on link $j$ is

$$
\operatorname{Pr}\left\{Z_{R}=l \mid X_{j}=m\right\}=\left(\begin{array}{c}
C \\
l
\end{array}\right) h_{l}\left(X_{j}=m\right) .
$$

We are now in a position to analyze the two routing schemes referred to earlier in the section. In fixed alternate routing (FAR), possibly the simplest and most widely used scheme, an ordered set of routes is assigned to each source-destination pair. When a call arrives, the RAW algorithm searches the routes in the prescribed order and assigns the first route which has a free wavelength. In case the route is an alternate route, it must have more free wavelengths than its reservation parameters before it can be assigned. For simplicity, we assume that all alternate routes have the same reservation parameter $r$.

Let $D_{S}$ denote the direct route for node pair $S$. Let $\mathcal{A}_{\mathcal{S}}$ denote the set of alternate routes for node pair $S$. This excludes the direct route. We assume that the alternate routes are link disjoint, and, hence, blocking on alternate routes is independent. This assumption is not very unrealistic since it is desirable to have link-disjoint alternate routes to overcome link failures, whenever possible. In case paths overlap, the approximation presented in [18] to consider the case of overlaps is directly applicable here. Since our main thrust is to show that our approach is applicable to alternate routing, we assume in this work that alternate paths are link-disjoint.

According to the above routing scheme, the set-up rate $\alpha_{j}(m)$ at link $j$, given exactly $m$ wavelengths are free is calculated as

$$
\begin{aligned}
\alpha_{j}(m)= & \sum_{S: j \in D_{S}} \lambda_{S} \operatorname{Pr}\left\{Z_{D_{S}}>0 \mid X_{j}=m\right\}, \\
\text { if } m \leq & r . \\
= & \sum_{S: j \in D_{S}} \lambda_{S} \operatorname{Pr}\left\{Z_{D_{S}}>0 \mid X_{j}=m\right\} \\
& +\sum_{S} \sum_{\substack{R: R \in \mathcal{A}_{\mathcal{S}} \\
j \in R}} \gamma_{R}^{S}(m) \operatorname{Pr}\left\{Z_{R}>r \mid X_{j}=m\right\},
\end{aligned}
$$$$
\text { if } m>r
$$

where $\gamma_{R}^{S}(m)$ is the traffic offered to alternate route $R$ by node pair $S$ given exactly $m$ wavelengths are free on link $j$.

From the FAR scheme, the traffic offered to an alternate route $R$ of node pair $S$ is the overflow traffic resulting from the previous $R-1$ routes being blocked. We assume this traffic to be Poisson, which is a reasonable assumption when the number of 
routes is small. The overflow traffic to route $R$ from a node pair $S$ may be calculated as

$\gamma_{R}^{S}(m)=\lambda_{S} \operatorname{Pr}\left\{Z_{D_{S}}=0, Z_{1} \leq r, \ldots, Z_{R-1} \leq r \mid X_{j}=m\right\}$

where $\{1, \ldots, R\} \in \mathcal{A}_{\mathcal{S}}$. Since we assume that the alternate routes block independently, we have

$$
\begin{aligned}
\gamma_{R}^{S}(m)=\lambda_{S} \operatorname{Pr}\left\{Z_{D_{S}}=\right. & \left.0 \mid X_{j}=m\right\} \\
& \cdot \prod_{T=1}^{R-1} \operatorname{Pr}\left\{Z_{T} \leq r \mid X_{j}=m\right\} .
\end{aligned}
$$

The probability that a direct route is blocked is given by

$$
\begin{aligned}
\operatorname{Pr}\left\{Z_{D_{S}}=0\right\} & =h_{0}^{D_{S}}, \\
\operatorname{Pr}\left\{Z_{D_{S}}=0 \mid X_{j}=m\right\} & =h_{0}^{D_{S}}\left(X_{j}=m\right) .
\end{aligned}
$$

The probability that an alternate route is blocked is given by

$$
\begin{gathered}
\operatorname{Pr}\left\{Z_{R} \leq r\right\}=\sum_{l=0}^{r}\left(\begin{array}{c}
C \\
l
\end{array}\right) h_{l}^{R}, \\
\operatorname{Pr}\left\{Z_{R} \leq r \mid X_{j}=m\right\}=\sum_{l=0}^{r}\left(\begin{array}{c}
C \\
l
\end{array}\right) h_{l}^{R}\left(X_{j}=m\right)
\end{gathered}
$$

where either expression may be used, depending on whether link $j$ lies on the alternate route or not. Also,

$$
\operatorname{Pr}\left\{Z_{R}>r\right\}=1-\operatorname{Pr}\left\{Z_{R} \leq r\right\} .
$$

Finally, the probability that a call for node pair $S$ is blocked is given by

$$
B_{S}=\operatorname{Pr}\left\{Z_{D_{S}}=0\right\} \prod_{R \in \mathcal{A}_{\mathcal{S}}} \operatorname{Pr}\left\{Z_{R} \leq r\right\}
$$

\section{A. Algorithm for Computing Blocking Probabilities}

We describe below, the algorithm for computing blocking probabilities in the network with fixed alternate routing by the method of iteration. We assume that the variable $g_{i}$ was already computed using methods given in Sections II-IV.

1) Initialization. Let $\hat{B}_{S}=0 \forall S$.

Let $\alpha_{j}(0)=0, \alpha_{j}(m)=\sum_{S: j \in D_{S}} \lambda_{j}, m=1, \ldots, C$.

2) Determine $q_{j}(\cdot), j=1, \ldots, J$, using (2) and (3).

3) Calculate $\alpha_{j}(\cdot), j=1, \ldots, J$ using (33)-(41).

4) Calculate $B_{S}$ for all node pairs, using (44).

If $\max _{S}\left|B_{S}-\hat{B}_{S}\right|<\epsilon$ then terminate. Else, let $\hat{B}_{S}=$ $B_{S}$, go to step 2 .

\section{B. Algorithm for Computing $\alpha_{j}(\cdot)$}

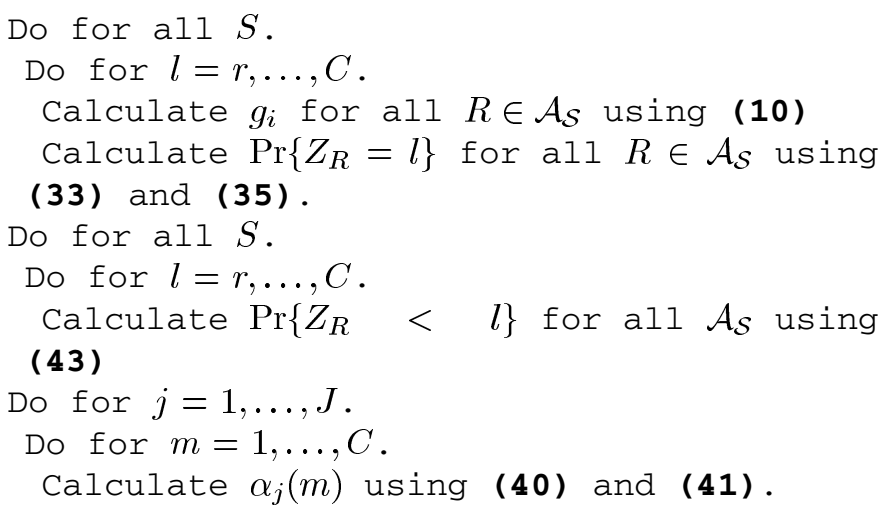

\section{LEAST LOADED ROUTING}

We analyze LLR for fully connected networks in this section. Such a scheme has been analyzed extensively for networks with at most two hops in [10] and also in [2]. Our method follows that of [2]. Each node pair has a direct link $\{j\}$ and a set of alternate routes denoted by $\mathcal{A}_{j}$. Hence, each node pair may be identified by its direct link. When a call arrives for a node pair say $\{j\}$, it is set up on the direct link if the number of free wavelengths on the link, $m_{j}>0$. Otherwise, the RWA algorithm attempts to assign the call to the alternate route with the largest number of free wavelengths. We assume, as in the case of FAR, that all alternate routes have a reservation parameter $r$, so that a call may be set up on an alternate route only if it has at least $r+1$ free wavelengths. In case of a tie, the routes in $\mathcal{A}_{j}$ are assumed to be ordered in some fashion, and the route with the smaller index is chosen. Denote by $\mathcal{S}_{j}$, the set of links (node pairs) adjacent to link (node pair) $j$. For a link (node pair) $j$ denote by alt $(j)$ the alternate route chosen according to the LLR scheme described above.

Based on the RWA algorithm described above, the set-up rate $\alpha_{j}(m)$ on link $j$ given exactly $m$ wavelengths are idle is calculated as follows:

$$
\begin{aligned}
\alpha_{j}(m) & =0, \quad \text { if } m=0 \\
& =\lambda_{j}, \quad \text { if } m \leq r \\
& =\lambda_{j}+\sum_{k: k \in \mathcal{S}_{j}} \lambda_{k} q_{k}(0) h\left(j, k_{R}, m\right), \quad \text { if } m>r
\end{aligned}
$$

where

$$
\begin{aligned}
h\left(j, k_{R}, m\right) & =\operatorname{Pr}\left\{\operatorname{alt}(k)=k_{R} ; Z_{k_{R}}>r \mid X_{j}=m\right\} \\
m & >r, \quad k_{R}: j \in k_{R}
\end{aligned}
$$

is the probability that node pair $k$ chose the alternate route $k_{R}$ and there are at least $r+1$ wavelengths free on route $k_{R}$ given that exactly $m$ wavelengths are idle on link $j \in k_{R}$. Using the chain rule and conditioning on disjoint events $\left\{Z_{k_{R}}=l: l=\right.$ $r+1, \ldots, m\}$, we can calculate $h\left(j, k_{R}, m\right)$ as follows

$$
\begin{aligned}
h\left(j, k_{R}, m\right)= & \sum_{l=r+1}^{m} \operatorname{Pr}\left\{Z_{k_{R}}=l \mid X_{j}=m\right\} \\
& \cdot \operatorname{Pr}\left\{\operatorname{alt}(k)=k_{R} \mid Z_{k_{R}}=l, X_{j}=m\right\} \\
= & \sum_{l=r+1}^{m} f\left(j, k_{R}, m, l\right) g\left(j, k_{R}, l\right)
\end{aligned}
$$

where

$$
\begin{aligned}
f\left(j, k_{R}, m, l\right) & =\operatorname{Pr}\left\{Z_{k_{R}}=l \mid X_{j}=m\right\} \\
& =\left(\begin{array}{c}
C \\
l
\end{array}\right) h_{l}\left(X_{j}=m\right)
\end{aligned}
$$

and

$$
g\left(j, k_{R}, l\right)=\operatorname{Pr}\left\{\text { alt }(k)=k_{R} \mid Z_{k_{R}}=l\right\} .
$$

Note that $g\left(j, k_{R}, l\right)$ is the probability that node pair $k$ choses alternate route $k_{R}$ given that there are $l$ wavelengths free on that route. This is independent of the event $X_{j}=m$ and is simply the probability that routes preceding $k_{R}$ have less than $l$ wavelengths free and those following $k_{R}$ have at most $l$ wavelengths 
free. The expression for $g\left(j, k_{R}, l\right)$ can be calculated in accordance with the LLR scheme as

$$
\begin{aligned}
g\left(j, k_{R}, l\right) & =\operatorname{Pr}\left\{\text { alt }(k)=k_{R} \mid Z_{k_{R}}=l\right\} \\
& =\prod_{k_{T} \in \mathcal{A}_{k}\left(k_{R}\right)^{-}} \operatorname{Pr}\left\{Z_{k_{T}}<l\right\} \prod_{k_{T} \in \mathcal{A}_{k}\left(k_{R}\right)^{+}} \operatorname{Pr}\left\{Z_{k_{T}} \leq l\right\}
\end{aligned}
$$

where $\mathcal{A}_{k}^{-}\left(k_{R}\right)$ denotes the set of routes in $\mathcal{A}_{k}$ preceding $k_{R}$ and $\mathcal{A}_{k}^{+}\left(k_{R}\right)$ is the set of routes following $k_{R}$ in the the assumed ordering. The probabilities in the above equation may be calculated using (43) which was derived in Section VII.

The probability that a node pair $j$ is blocked is given by

$$
B_{j}=\operatorname{Pr}\left\{X_{j}=0\right\} \prod_{R \in \mathcal{A}_{j}} \operatorname{Pr}\left\{Z_{R} \leq r\right\} .
$$

We reproduce below an algorithm originally proposed in [2] for computing the blocking probabilities in a network with LLR.

\section{A. Algorithm for Computing Blocking Probabilities}

1) Initialization. Let $\hat{B}_{j}=0, j=1, \ldots, J$. Let $\alpha_{j}(0)=0, \alpha_{j}(m)=\lambda_{j}, m=1, \ldots, C$.

2) Determine $q_{j}(\cdot), j=1, \ldots, J$, using (2) and (3).

3) Calculate $\alpha_{j}(\cdot), j=1, \ldots, J$ using (45).

4) Calculate $B_{j}$ for all node pairs, using (51). If $\max _{j}\left|B_{j}-\hat{B}_{j}\right|<\epsilon$ then terminate. Else, let $\hat{B}_{j}=$ $B_{j}$, go to step 2 .

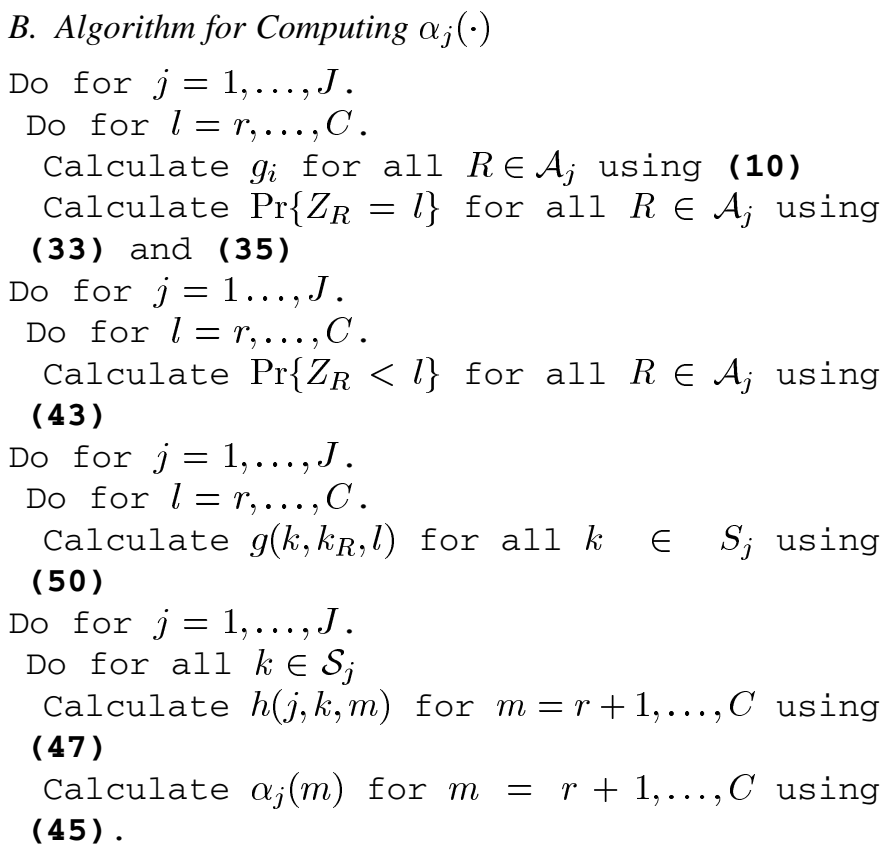

\section{RESULTS AND NUMERICAL COMPUTATION}

In this section we analyze the complexity of the techniques presented in this work and also examine their accuracy by applying them to various topologies under different traffic patterns.

\section{A. Complexity}

One of the main aims of this paper was to propose analytical models with reduced complexity to enable the study of large net-
TABLE I

TIME COMPLEXITY OF THE THREE MODELS FOR THE ARPA-2 AND 6-Node Ring Network. All Times are IN SECONDS, AND $C$ REFERS TO THE CAPACITY OF EACH LINK

\begin{tabular}{c|c|c|c}
\hline Network & $\begin{array}{c}\text { Birman's } \\
\text { Model } \\
\text { (secs) }\end{array}$ & $\begin{array}{c}\text { Indepe- } \\
\text {-ndence } \\
\text { (secs) }\end{array}$ & $\begin{array}{c}\text { Corr- } \\
\text {-elation } \\
\text { (secs) }\end{array}$ \\
\hline Ring, $C=8$ & 3.89 & 0.06 & 0.09 \\
\hline Ring, $C=16$ & 69.72 & 0.29 & 0.42 \\
\hline ARPA-2, $C=8$ & 263.02 & 0.29 & 1.73 \\
\hline ARPA-2, $C=16$ & $2.02 \times 10^{4}$ & 1.73 & 3.26 \\
\hline
\end{tabular}

works. We now study the complexity of the various techniques presented in this paper and also compare them against those presented in [2].

The state probabilities $q_{j}(m)$ (2) require $O(C)$ computation for each link. The $\beta$ coefficients (6) require $O(C)$ computation for each link and the path blocking computation complexity is $O(H C)$ (9) and (10). For the correlation model, the only difference is that the path blocking computation complexity is $O\left(H C^{2}\right)$. To the best of the authors' knowledge, these techniques are simpler with lower computation overhead than any of the existing models with the exception of [1] which was formulated for static scenarios only. In spite of the simplicity, as we shall observe, our results are quite accurate.

The complexity of computation of route blocking for the technique presented in [2] is $O\left(C^{H}\right)$ for fixed routing, which limits its applicability to small dense networks. We highlight this computational advantage by presenting results of time taken for computation for two networks, the 6-node ring and the 21-node ARPA-2 network, in Table I. All computations were done on a Sun Ultra SPARC system running at $150 \mathrm{MHz}$. The maximum hop length in the 6-node ring was 3, and for the ARPA-2 network, it was limited to 4 . As can be seen from the results, the Independence and Correlation Models are far superior than [2] in terms of time complexity. Although, not explicitly evident, the Independence model gives exactly the same results as the model in [2]. This is because both models make the same assumptions, and calculate the number of free wavelengths correctly, albeit in different ways, under these assumptions.

The computation requirements for FAR are $O\left(S R C^{2}\right)+$ $O(J S R C)$ where $S$ is the total number of node pairs and $R$ is the average number of routes for each node pair. This assumes that computations are done using the Independence Model. LLR computations requirements are similar to those required in [2] because only two hops were considered, although our analysis can clearly be extended to larger number of hops without significant increase in complexity.

\section{B. Numerical Results}

We now present results of both our techniques for a variety of topologies and compare them against simulations to study their accuracy. Our goal is to demonstrate the effectiveness of the models in terms of scalability and accuracy. For fixed routing, four topologies were chosen, a 6-node ring, a 12-node ring, a 


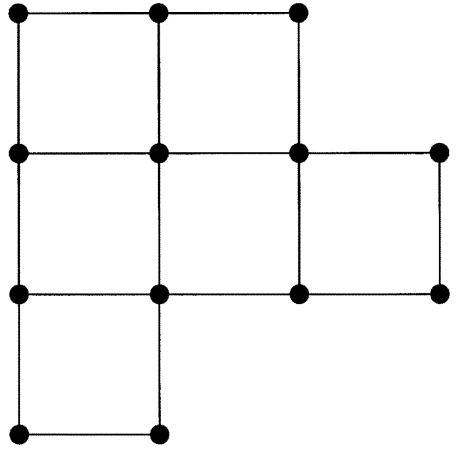

Fig. 1. A 13-node 18 link mesh network.

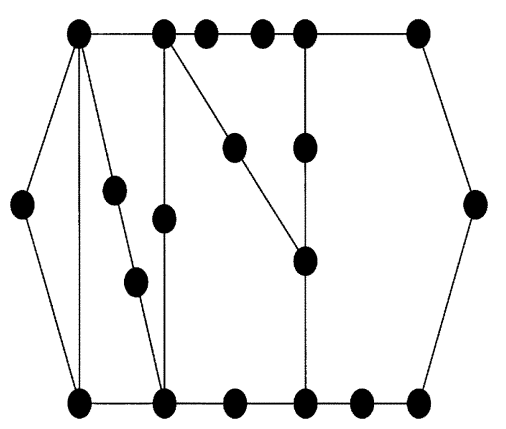

Fig. 2. The 21-node 26 Link ARPA-2 network.

13-node mesh network (Fig. 1), and the 21-node ARPA-2 network (Fig. 2). The ring networks were chosen to study the efficacy of our methods when applied to sparse networks, and the mesh and ARPA-2 were chosen as examples of two arbitrary topologies. Calculations were done for 16 and 32 wavelengths for all topologies. For the ARPA-2 and 12-node ring networks, we also compute blocking probabilities with 64 wavelengths. The maximum hop length was restricted to 3 for the 6-node ring, 6 for the 12-node ring, and 5 for the 13-node mesh and the 21-node ARPA-2 network. Routes were constructed using minimum hop routing and number 18 in the 6-node ring, 72 in the 12-node ring, 73 in the mesh network, and 76 routes in the ARPA-2 network. For the ring networks, a single path was chose for each possible node pair (unordered). For the 13-node mesh and ARPA-2 network, node pairs were chosen randomly and when multiple paths between node pairs were present, at most three were randomly selected, in which case the traffic was split equally over all paths. The accuracy of our models were studied under three different traffic patterns. They can be compactly written by the equation

$$
T_{H}=q^{H-1} \cdot T_{1}
$$

where $T_{i}$ is the traffic on a $i$-hop path. Three values of $q$ were chosen:

- $q=1.0$ uniform traffic;

- $q=0.5$ traffic dominated by smaller hop routes (low correlation);

- $q=1.5$ traffic dominated by larger hop routes (significant correlation).

By varying the parameter $q$ we can cover the range of link load correlation introduced by the wavelength continuity constraint. For simulations, 400000 calls were taken in each batch and 20

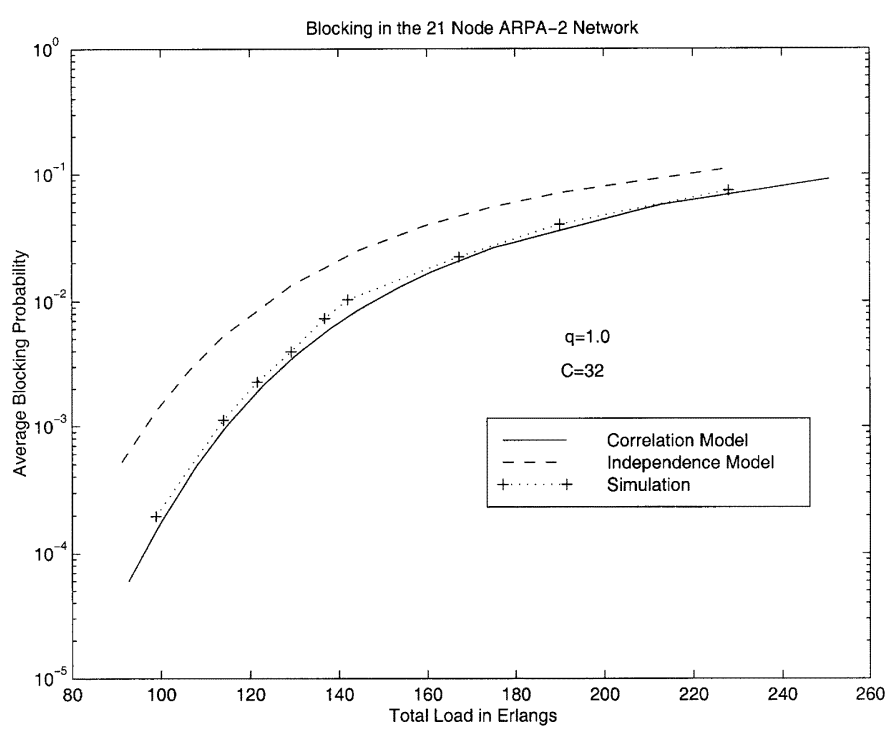

Fig. 3. Plot showing average blocking probability of the ARPA-2 network for $C=32$ and uniform traffic $(q=1.0)$.

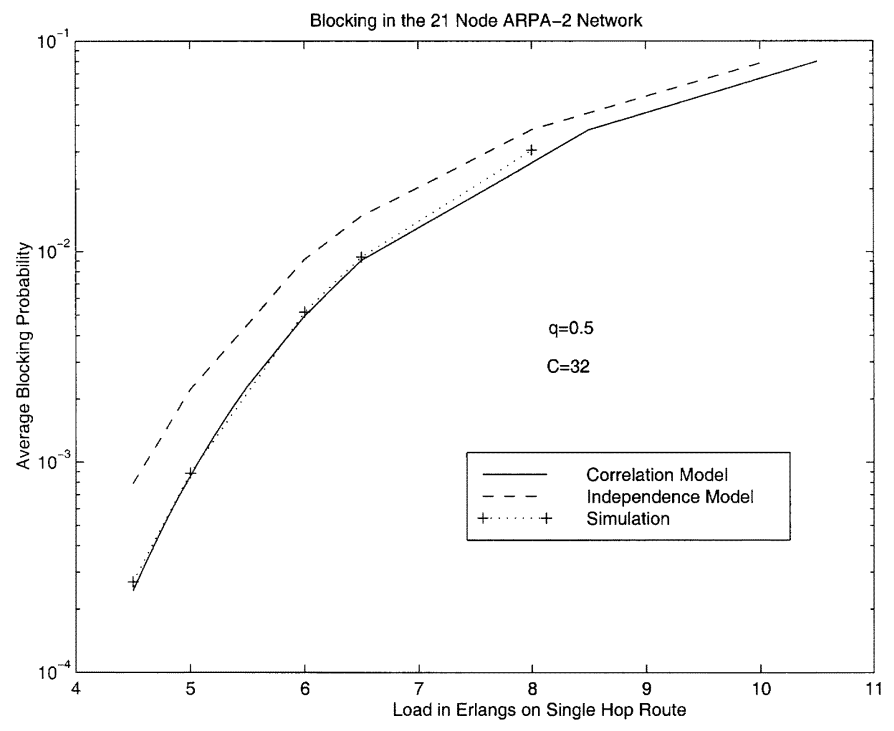

Fig. 4. Plot showing average blocking probability of the ARPA-2 network for $C=32$ and nonuniform traffic $(q=0.5)$.

batches were run for each load, The data points reported are in the $95 \%$ confidence interval. When using the iterative algorithm for analysis, iterations were stopped when blocking estimates in successive steps differed by less than $\epsilon=10^{-6}$.

\section{Fixed Routing}

We now discuss our results for each traffic pattern and network. For fixed routing, we present results only for 32 and 64 wavelengths. Results for other networks and wavelengths under the different traffic patterns are similar and the observations we make are valid for them also. Hence, we do not repeat these figures here.

For the ARPA-2 network (Fig. 3) and the 13-node mesh network (Fig. 7) we see that the Independence Model gives reasonable estimates to the blocking probability for uniform traffic $(q=1.0)$. The estimates obviously improve when the multi-link traffic is less $(q=0.5)$ as shown for the ARPA-2 network 


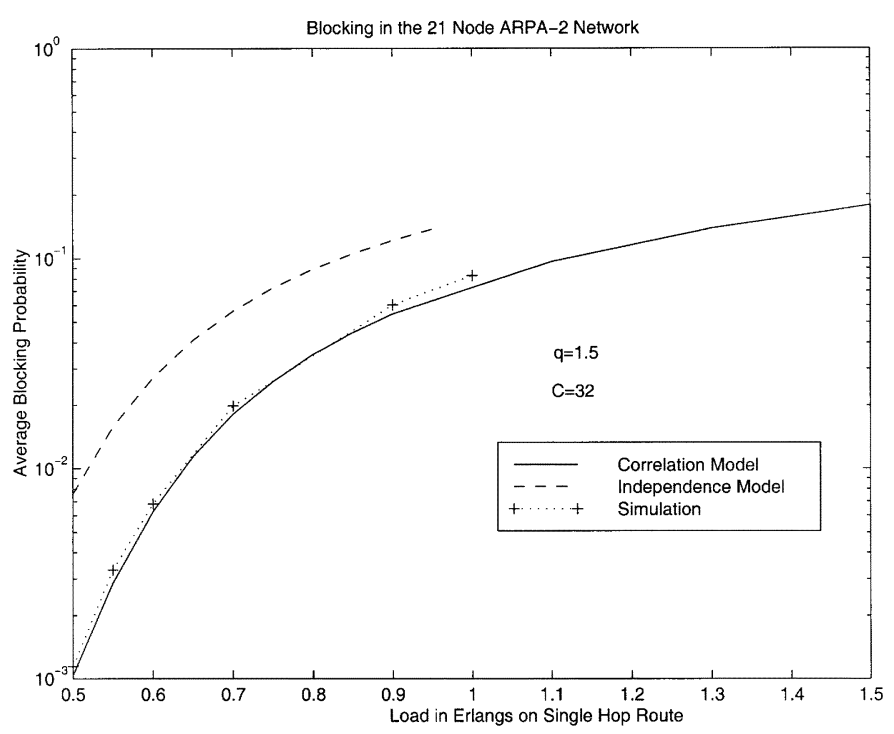

Fig. 5. Plot showing average blocking probability of the ARPA-2 network for $C=32$ and nonuniform traffic $(q=1.5)$.

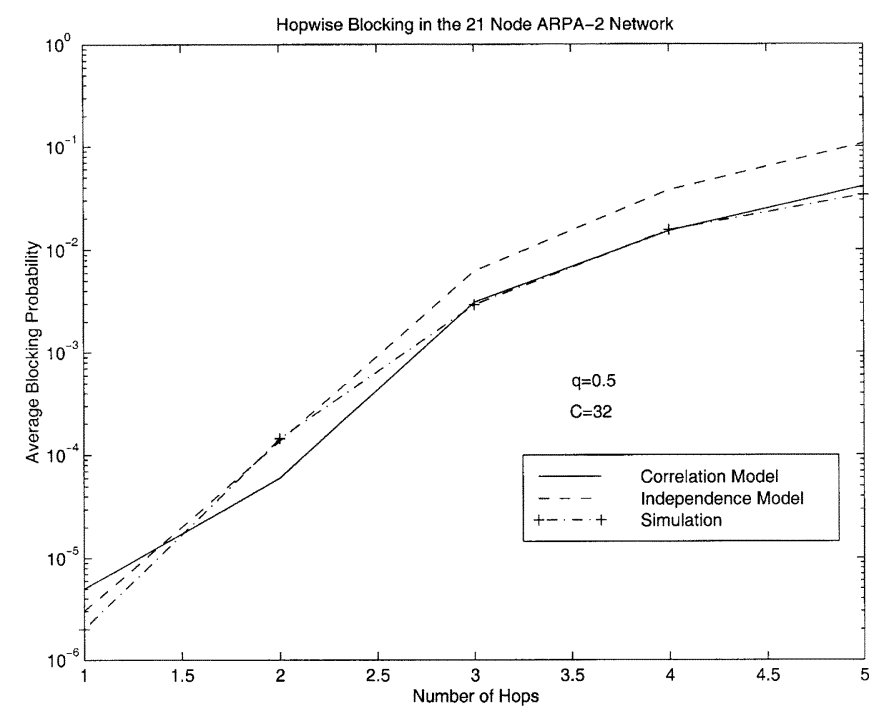

Fig. 6. Plot showing hop-wise blocking probability of the ARPA-2 network for $C=32$ and nonuniform traffic $q=0.5$.

in Fig. 4 because of lower correlation. However, when correlation increases $(q=1.5)$, the results of the Independence model degrade for the ARPA-2 network (Fig. 5) because the approximation that sets of wavelengths on adjacent links are independent is no longer a good one. The Correlation Model is seen to give fairly good results for both these topologies under all traffic conditions as can be expected from the original formulation. The accuracy of the correlation model can be seen from the hop wise blocking probability plotted in Figs. 6 and 8 for the ARPA-2 and 13-node mesh network, respectively.

Results of the 6-node and 12-node ring networks accentuate this the difference between the Correlation and the Independence Model in handling wavelength correlation. The Independence model overestimates the blocking for the 6-node ring (Fig. 9) as well as the 12-node ring (Fig. 10) though the results are not too bad for the 6-node ring because of the short paths. The results improve only marginally for the 12-node ring under

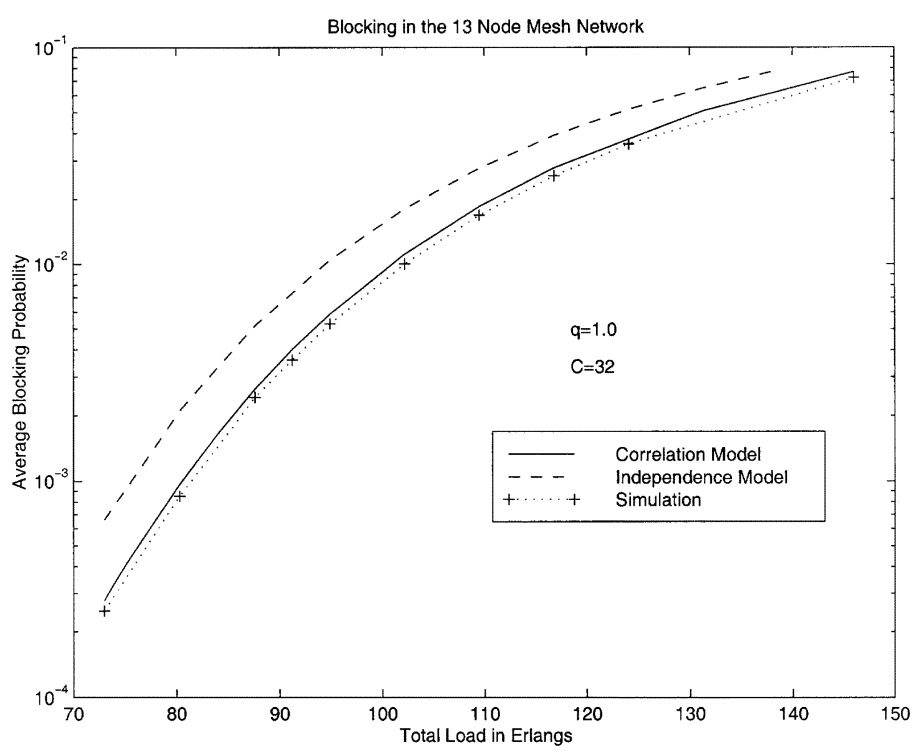

Fig. 7. Plot showing average blocking probability of the 13-node mesh network for $C=32$ and uniform traffic $(q=1.0)$.

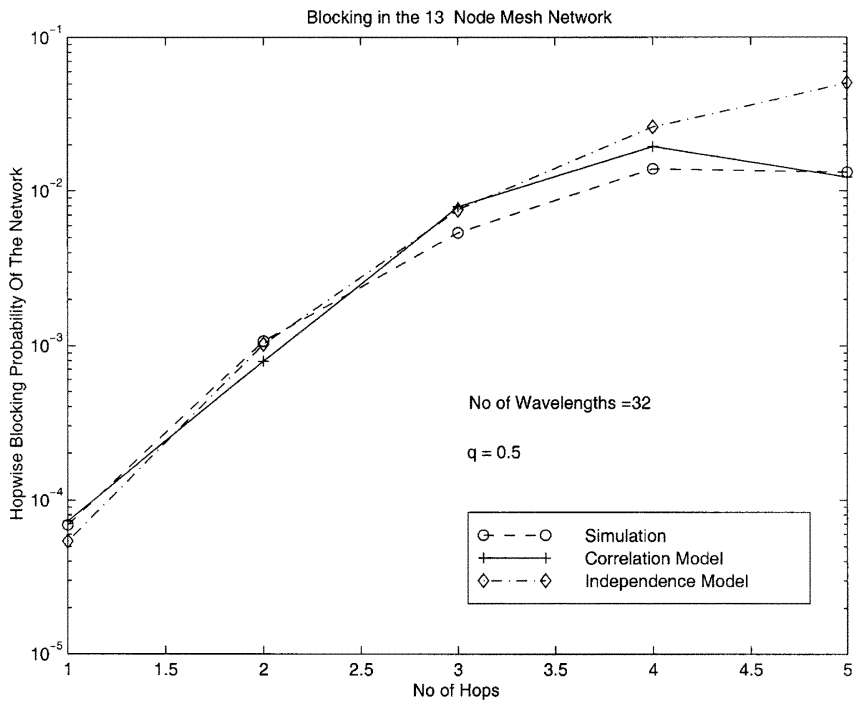

Fig. 8. Plot showing hop-wise blocking probability of the 13-node mesh network for $C=32$ and nonuniform traffic, $q=0.5$.

nonuniform traffic with reduced correlation $(q=0.5)$ (Fig. 11) and the estimates are off by more than two orders of magnitude when the correlation increases $(q=1.5)$ (Fig. 12) confirming results of previous researchers that sparse networks introduce significant wavelength correlation. The accuracy of the Correlation Model in handling this correlation is confirmed by application to such networks. Under all traffic patterns for both the ring networks it is seen to give reasonable estimates. We, hence, conclude that the Independence Model gives fair estimates for topologies which are well connected and have traffic patterns that result in low to medium correlation, while the Correlation Model may be used on a wide variety of networks even when connectivity is sparse and traffic patterns induce large correlation. To check the effectiveness of our models when the capacity of the link increases, we present blocking probability computations for the ARPA-2 Network (Fig. 13) and 12-node ring (Fig. 14) with 64 wavelengths per link and uniform traffic. Due 


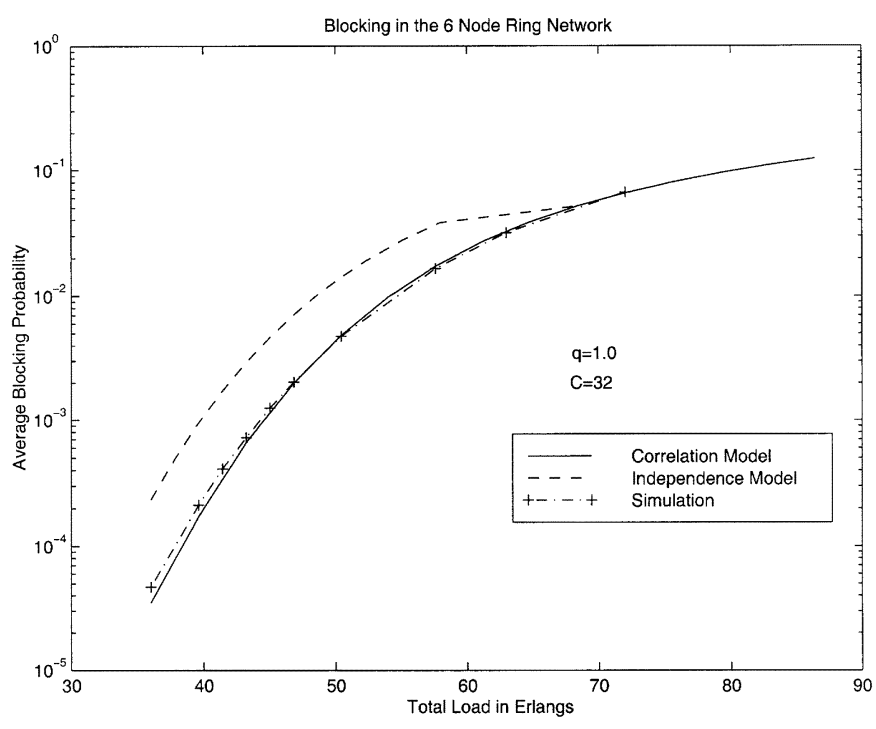

Fig. 9. Plot showing average blocking probability of the 6-node ring network for $C=32$ and uniform traffic $q=1.0$.

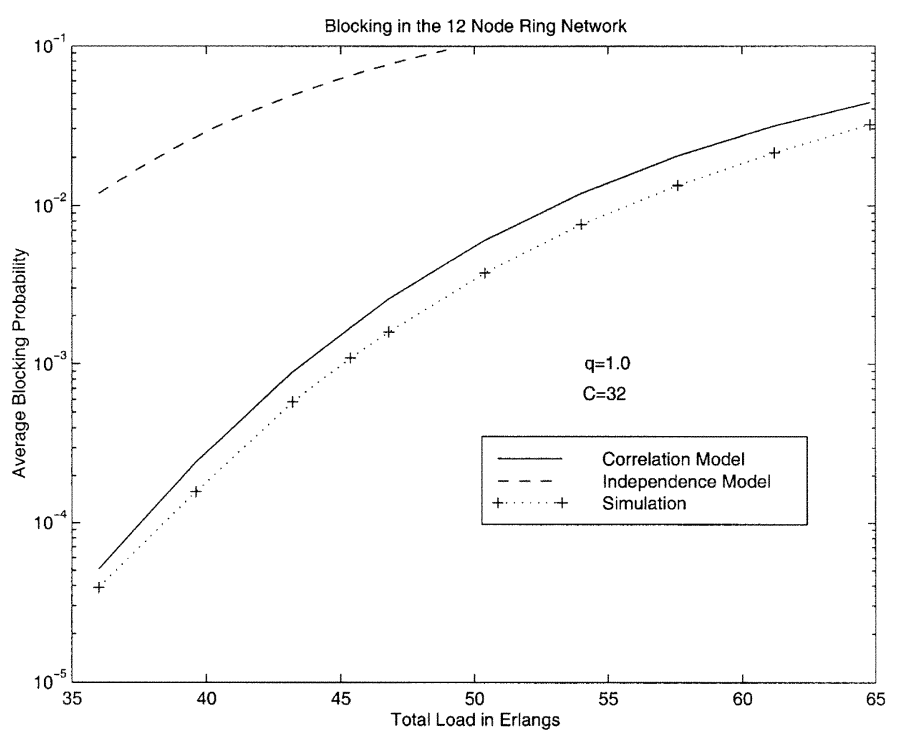

Fig. 10. Plot showing average blocking probability of the 12-node ring network for $C=32$ and uniform traffic $(q=1.0)$.

to the large number of wavelengths, the combinatorial terms can introduce round off errors. We removed such errors by ignoring the extremely small blocking probabilities as well as negative blocking probabilities since they would have been caused by round off errors in extremely small values and as such, do not impact the final solution much. The figures show that our models follow the simulation reasonably closely, demonstrating the scalability of the model.

\section{Alternate Routing}

For FAR, we have chosen the 6-node ring with 16 wavelengths. Each node pair has a direct and an alternate route, and there are 18 such node pairs. We present results of only the Independence Model and simulations in Figs. 15 and 16 since we are still researching the problem of estimating the correlation coefficients for the Correlation Model under a routing scheme

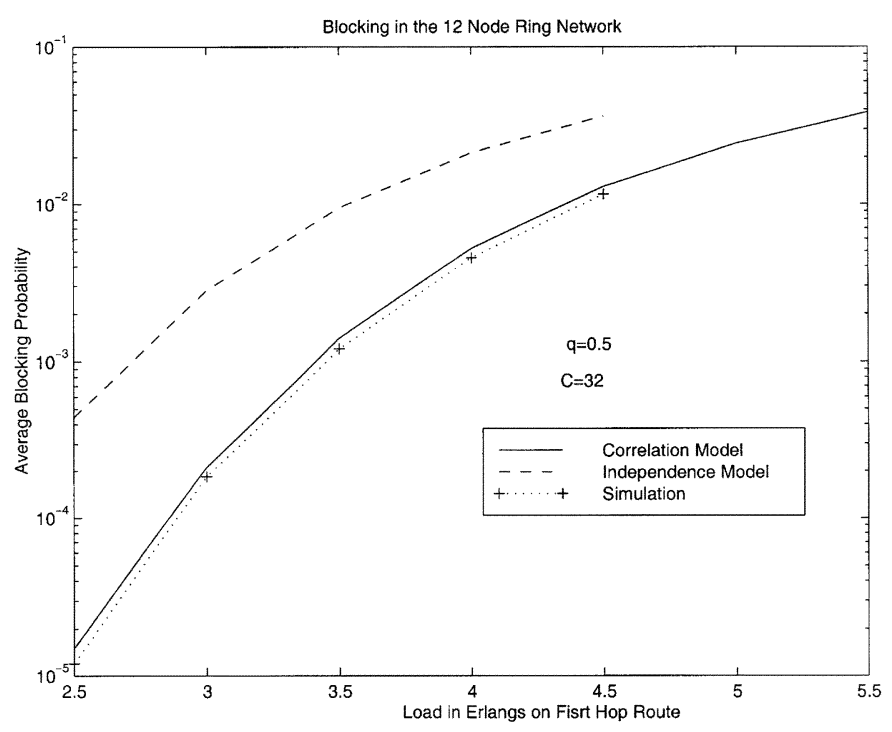

Fig. 11. Plot showing average blocking probability of the 12-node ring network for $C=32$ and nonuniform traffic $(q=0.5)$.

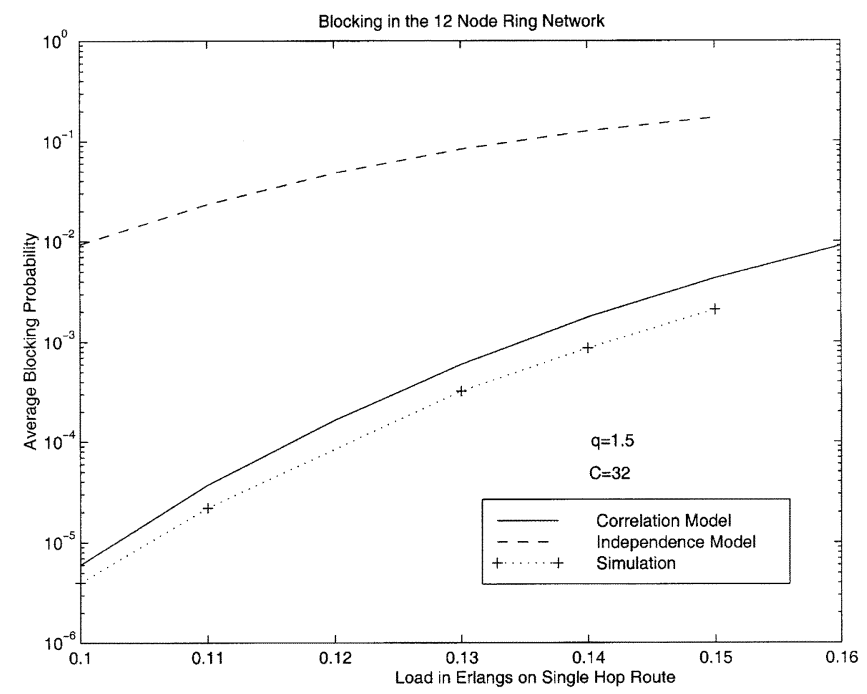

Fig. 12. Plot showing average blocking probability of the 12-node ring network for $C=32$ and nonuniform traffic $(q=1.5)$.

apart from fixed routing. Ad hoc extension of the method explained in Section IV to FAR or LLR leads to an underestimation of the blocking in the network. As of now, we do not know why this is so. We note from Figs. 15 and 16 that the model provides a good approximation at lower blocking probabilities, which is the important region when considering problems like dimensioning of the network. LLR was studied on a 4-node fully connected network with 16 wavelengths and uniform traffic. Results are shown in Fig. 17 for the Independence Model computations and simulations. For least loaded routing, our model is very accurate, closely following the simulation results.

\section{CONCLUSIONS}

We have proposed two analytical techniques of low complexity, the Independence Model and the Correlation Model, for the study of wavelength routed networks with arbitrary topology and traffic patterns. Both models involve only simple 


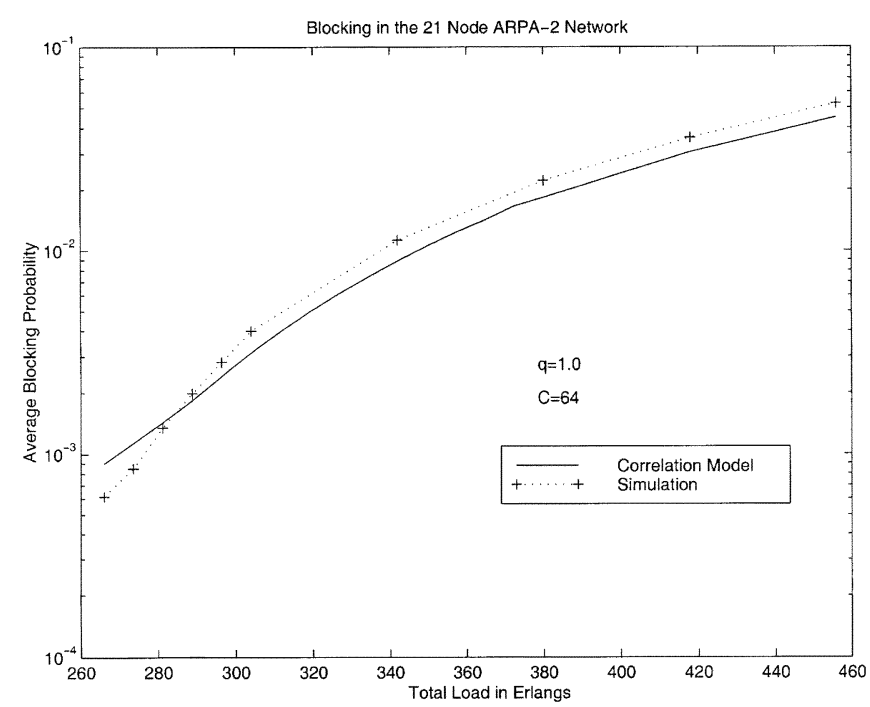

Fig. 13. Blocking in the ARPA-2 network with $C=64$ and uniform traffic $(q=1.0)$.

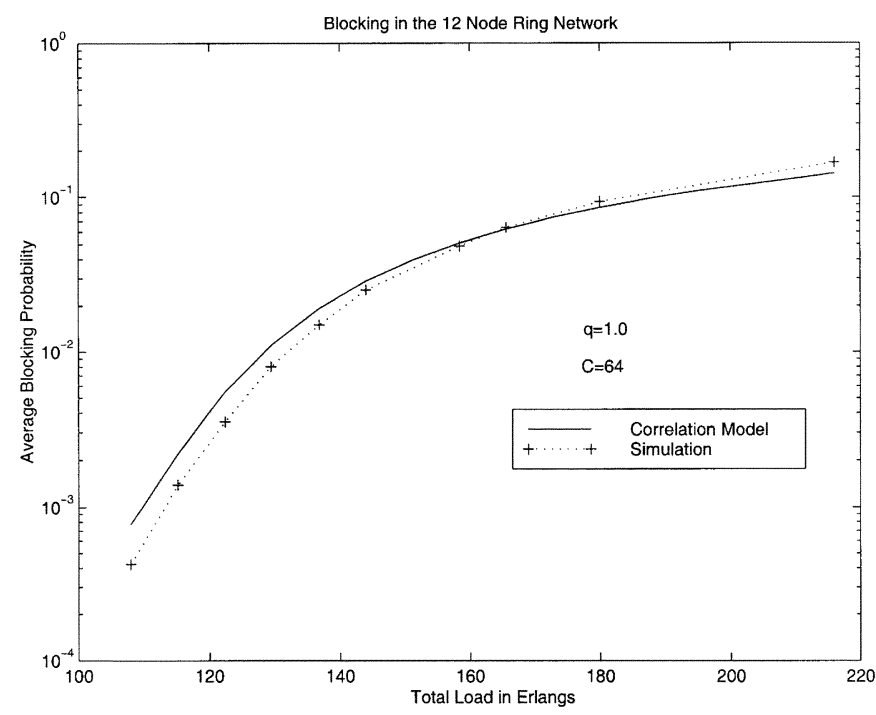

Fig. 14. Blocking in the 12-node ring network with $C=64$ and uniform traffic $(q=1.0)$.

computations and can be used for fast calculations of blocking probabilities. Through computations we have shown that the Independence Model gives good estimates when the network is well connected and the Correlation Model is accurate even for sparse networks under fixed routing. The simplicity of our models can be exploited when computing blocking probabilities in reasonably large networks with a large number of wavelengths as shown in this paper for the ARPA-2 and 12-node ring networks with 64 wavelengths. The Correlation Model, however, is not insensitive to the direction in which we proceed along a route when we compute the blocking and may lead to incorrect results under highly skewed traffic patterns. Nevertheless, we believe such events are unlikely to happen in practice and that the model is quite useful in obtaining good and quick estimates of blocking that would be beneficial in computations to dimension networks. We have also extended the Independence Model to study Fixed Alternate Routing and

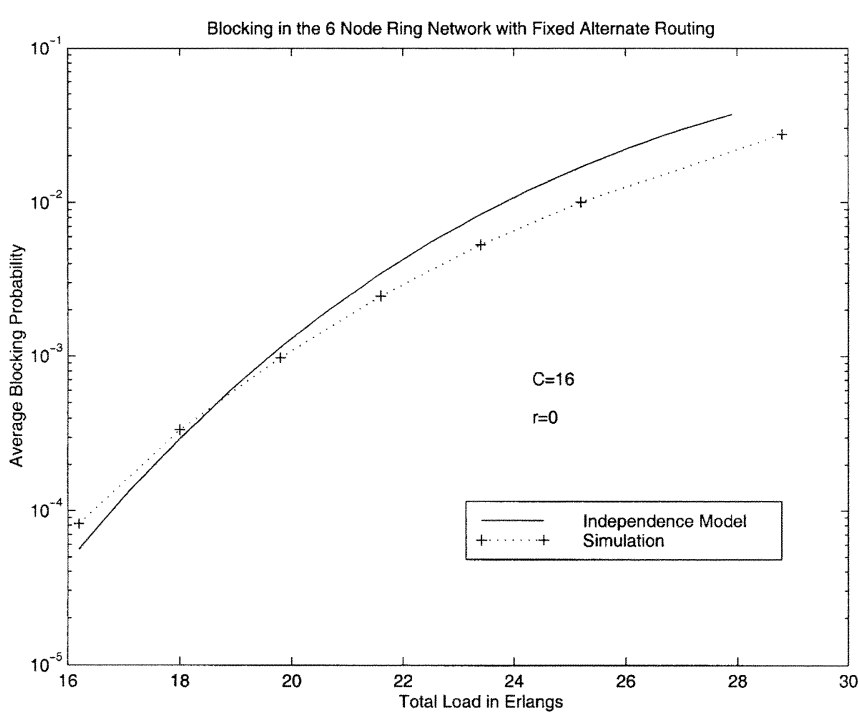

Fig. 15. Plot showing average blocking probability of the 6-node ring network for $C=16$, and Fixed Alternate Routing $(r=0)$.

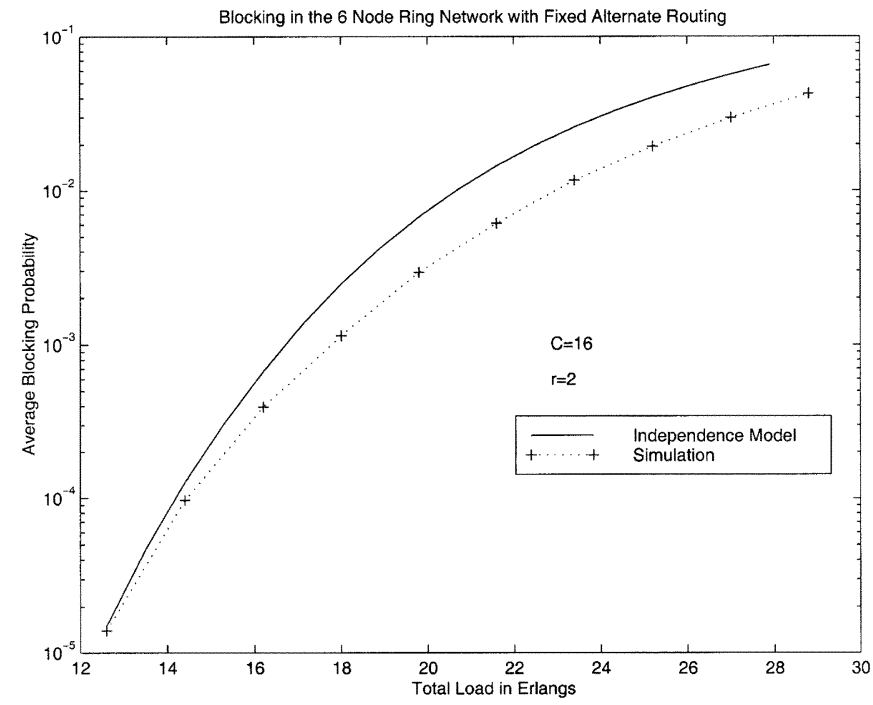

Fig. 16. Plot showing average blocking probability of the 6-node ring network for $C=16$, and fixed alternate routing $(r=2)$.

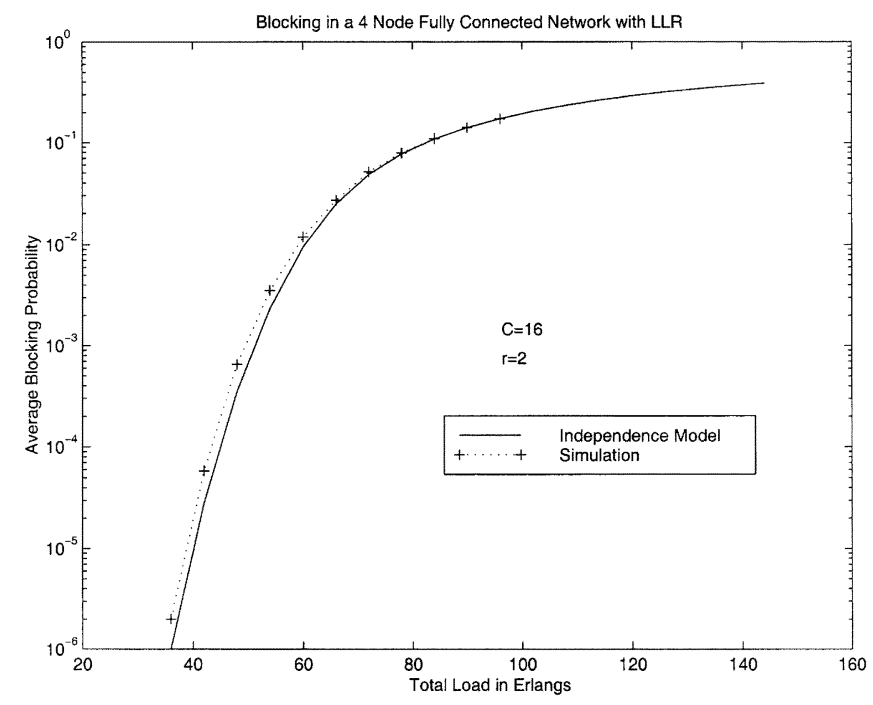

Fig. 17. Plot showing average blocking probability of the 4-node network for $C=16$, uniform traffic and least loaded routing $(r=2)$. 
Least Loaded Routing and found it to give good results in the region of interest, i.e., low blocking probability.

\section{REFERENCES}

[1] R. A. Barry and P. A. Humblet, "Models of blocking probability in alloptical networks with and without wavelength changers," IEEE J. Select. Areas Commun., vol. 14, pp. 867-878, June 1996.

[2] A. Birman, "Computing approximate blocking probabilities for a class of all-optical networks," IEEE J. Select. Areas Commun., vol. 14, pp. 852-857, June 1996.

[3] E. Mannie et al., "Generalized multi-protocol label switching (GMPLS) architecture," IETF, Internet Draft, draft-ietf-ccamp-gmpls-architecture07.txt, May 2003

[4] A. Girard, Routing and Dimensioning in Circuit-Switched Networks. Boston, MA: Addision-Wesley, 1990.

[5] M. Murata, H. Harai, and H. Miyahara, "Performance of alternate routing methods in all-optical switching networks," in Proc. IEEE INFOCOM, 1997, pp. 516-524.

[6] _ - "Performance analysis of wavelength assignment policies in alloptical networks with limited-range wavelength conversion," IEEE J. Select. Areas Commun., vol. 16, pp. 1051-1060, Sept. 1998.

[7] E. Karasan and E. Ayanoglu, "Performance of WDM transport networks," IEEE J. Select. Areas Commun., vol. 16, pp. 1081-1096, Sept. 1996.

[8] — "Effects of wavelength routing and selection algorithms on wavelength conversion gain in WDM optical networks," IEEE/ACM Trans. Networking, vol. 6, pp. 186-196, Apr. 1998.

[9] F. P. Kelly, "Routing and capacity allocation in networks with trunk reservation," Math. Oper. Res., vol. 15, pp. 771-792, 1990.

[10] K.-M. Chan and T.-S. P. Yun, "Analysis of least congested path routing in WDM lightwave networks," in Proc. IEEE INFOCOM, vol. 2, Mar. 1994, pp. 962-969.

[11] A. Mokhtar and M. A. Glu, "Adaptive wavelength routing in all-optical networks," IEEE/ACM Trans. Networking, vol. 6, pp. 197-206, Apr. 1998.

[12] B. Ramamurthy and B. Mukherjee, "Wavelength conversion in WDM networking," IEEE J. Select. Areas Commun., vol. 16, pp. 1061-1073, Sept. 1996.

[13] R. Ramaswami, "Multi-wavelength lightwave networks for computer communication," IEEE Commun. Mag., vol. 31, pp. 78-88, Feb. 1993.

[14] R. Ramaswami and K. N. Sivarajan, "Routing and wavelength assignment in all-optical networks," IEEE/ACM Trans. Networking, vol. 3, pp. 489-500, Oct. 1995.

[15] — Optical Networks: A Practical Perspective. San Fransisco, CA Morgan Kaufmann, 1998.

[16] M. Azizoglu, S. Subramanium, and A. Somani, "All-optical networks with sparse wavelength conversion," IEEE/ACM Trans. Networking, vol. 4, pp. 544-557, Aug. 1996.
[17] A. Kasper, S.-P. Chung, and K. Ross, "Computing approximate blocking probabilities for large loss networks with state-dependent routing," IEEE/ACM Trans. Networking, vol. 1, pp. 105-115, Feb. 1993.

[18] G. N. Rouskas, Y. Zhu, and H. G. Perros, "A path decomposition approach for computing blocking probabilities in wavelength-routing networks," IEEE/ACM Trans. Networking, vol. 8, pp. 747-762, Dec. 2000.

[19] —, "Blocking in wavelength-routing networks-Part I: The single-path case," in Proc. IEEE INFOCOM, Mar. 1999, pp. 321-328.

[20] _ _Blocking in wavelength-routing networks-Part II: Mesh topologies," in Proc. 16th Int. Teletrafic Congr., June 1999, pp. 1321-1330.

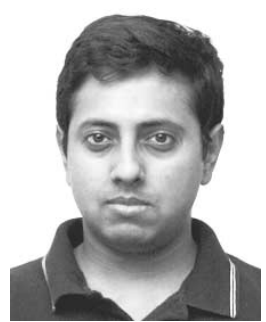

Ashwin Sridharan ( $\left.\mathrm{S}^{\prime} 00\right)$ received the Bachelor degree in electronic engineering from Regional Engineering College, Nagpur, India, in 1997 and the M.E. degree from the Indian Institute of Science, Bangaore, in 1999. He is currently pursuing the doctora degree at the University of Pennsylvania, Philadelphia.

His research interests include traffic engineering and routing algorithms with emphasis on enhancing their performance in existing frameworks.

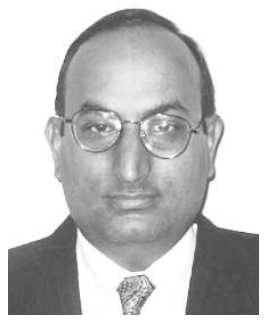

Kumar N. Sivarajan (M'88) was born on October 23, 1968, in Bangalore, India. He received the B.Tech degree in electrical engineering from the Indian Institute of Technology Madras, India, in 1987 and the M.S. and Ph.D. degrees in electrical engineering from the California Institute of Technology, Pasadena, in 1988 and 1990 , respectively.

From 1990 to 1994 he was with the IBM T. J. Watson Research Center, Yorktown Heights, NY. From 1994 to 2002 he was Associate Professor in the Electrical and Communication Engineering Department at the Indian Institute of Science, Bangalore, India. He is currently with TejasNetworks.

He was an Academic Visitor with the IBM T. J. Watson Research Center during the summers of 1996 and 1997. His research interests include modeling, design, and performance evaluation of cellular, optical, and ATM Networks.

Dr. Sivarajan is an Associate of the Indian Academy of Sciences. He is the recipient of the IEEE Charles LeGeyt Fortescue Fellowship for the academic year 1987-1988 and the corecipient with Dr. R. Ramaswami of the IEEE Communication Society 1996 William R. Bennett Prize Paper Award and the 1997 IEEE W. R. G. Baker Prize Paper Award. He is an Editor of the IEEE/ACM TRANSACTIONS ON NETWORKING. 